西

\title{
AN EXPLICIT VISCOSITY ITERATIVE ALGORITHM FOR FINDING THE SOLUTIONS OF A GENERAL EQUILIBRIUM PROBLEM SYSTEMS
}

\author{
H. R. SAHEBI AND S. EBRAHIMI
}

\begin{abstract}
We suggest an explicit viscosity iterative algorithm for finding a common element of the set of solutions for an general equilibrium problem system (GEPS) involving a bifunction defined on a closed, convex subset and the set of fixed points of a nonexpansive semigroup on another one in Hilbert's spaces. Furthermore, we present some numerical examples(by using MATLAB software) to guarantee the main result of this paper.
\end{abstract}

\section{Preliminaries}

Let $H$ be a real Hilbert space with norm $\|$.$\| and inner product \langle.,$.$\rangle Let C$ be a nonempty closed convex subset of $H$. Then, for any $x \in H$, there exists a unique nearest point in $C$, denoted by $P_{C} x$, such that

$$
\left\|x-P_{C} x\right\| \leq\|x-y\|, \text { forally } \in C .
$$

Such $P_{C}$ is called the metric projection of $H$ onto $C$. We know that $P_{C}$ is nonexpansive. The strongly (weak) convergent of $\left\{x_{n}\right\}$ to $x$ is written by $x_{n} \rightarrow x\left(x_{n}-x\right)$ as $n \rightarrow \infty$. Moreover, $H$ satisfies the Opial's condition [15], if for any sequence $\left\{x_{n}\right\}$ with $x_{n} \rightarrow x$, the inequality

$$
\liminf _{n \rightarrow \infty}\left\|x_{n}-x\right\|<\liminf _{n \rightarrow \infty}\left\|x_{n}-y\right\|
$$

holds for every $y \in H$ with $x \neq y$.

Recall that a mapping $T: C \rightarrow C$ is said to be nonexpansive, if $\|T x-T y\| \leq\|x-y\|$ for all $x, y \in C . F(T)$ denotes the set of fixed points of $T$. Let $\{T(s): s \in[0, \infty)\}$ be a nonexpansive semigroup on a closed convex subset $C$, that is,

Received May 29, 2014, accepted November25, 2014.

2010 Mathematics Subject Classification. Primary: 47H09, 47H10; Secondary: 47J20.

Key words and phrases. Nonexpansive semigroup, general equilibrium problems system, strongly positive linear bounded operator, $\alpha$-inverse strongly monotone mapping, fixed point, Hilbert space.

Corresponding author: H. R. Sahebi. 
(i) $T(0) x=x$, for all $x \in C$;

(ii) $T(s+t)=T(s) T(t)$, for all $s, t \geq 0$;

(iii) $\|T(s) x-T(s) y\| \leq\|x-y\|$, for all $x, y \in C$ and $s \geq 0$;

(iv) $s \mapsto T(s) x$ is continuous for all $x \in C$.

Denote by $F(S)=\bigcap_{s \geq 0} F(T(s))$. It is well known that $F(S)$ is closed and convex subset in $H$ and $F(S) \neq \varnothing$ if $C$ is bounded [1]. Recall that a self mapping $f: H \rightarrow H$ is a contraction if there exists $\rho \in(0,1)$ such that $\|f(x)-f(y)\| \leq \rho\|x-y\|$ for each $x, y \in H$.

A mapping $B: C \rightarrow H$ is called $\alpha$-inverse strongly monotone [14, 20] if there exists a positive real number $\alpha>0$ such that for all $x, y \in C$

$$
\langle B x-B y, x-y\rangle \geq \alpha\|B x-B y\|^{2} .
$$

Shimizu and Takahashi [18] studied the strongly convergent of the sequence $\left\{x_{n}\right\}$ which is defined by:

$$
x_{n+1}=\alpha_{n} x+\left(1-\alpha_{n}\right) \frac{1}{t_{n}} \int_{0}^{t_{n}} T(s) x_{n} d s, x \in C,
$$

in a real Hilbert space, where $\{T(s): s \in[0, \infty)\}$ is a strongly continuous semigroup of nonexpansive mappings on a closed convex subset $C$ of a Hilbert space and $\lim _{n \rightarrow \infty} t_{n}=\infty$. Later, Plubtieng and Punpaeng [17] introduced the following iterative method:

$$
x_{n+1}=\alpha_{n} f\left(x_{n}\right)+\beta_{n} x_{n}+\left(1-\alpha_{n}-\beta_{n}\right) \frac{1}{t_{n}} \int_{0}^{t_{n}} T(s) x_{n} d s,
$$

where $\left\{\alpha_{n}\right\},\left\{\beta_{n}\right\}$ are the sequences in $(0,1),\left\{s_{n}\right\}$ is a positive real divergent sequence and $f: C \rightarrow C$ is a contraction. Under the conditions $\sum_{n=1}^{\infty} \alpha_{n}=\infty, \alpha_{n}+\beta_{n}<1, \lim _{n \rightarrow \infty} \alpha_{n}=0$ and $\lim _{n \rightarrow \infty} \beta_{n}=0$, they proved the strong convergence of the sequence.

Also, Plubtieng and Punpaeng [16] introduced the following iterative scheme: Let $S: C \rightarrow H$ be a nonexpansive mapping, defined sequences $\left\{x_{n}\right\}$ and $\left\{u_{n}\right\}$ by

$$
\left\{\begin{array}{l}
F\left(u_{n}, y\right)+\frac{1}{r_{n}}\left\langle y-u_{n}, u_{n}-x_{n}\right\rangle \geq 0 ; \\
x_{n+1}=\alpha_{n} \gamma f\left(x_{n}\right)+\left(I-\alpha_{n} A\right) S u_{n}, \forall y \in H .
\end{array}\right.
$$

They proved, under the certain appropriate conditions, the sequence $\left\{x_{n}\right\}$ converges strongly to the unique solution of the variational inequality

$$
\langle(A-\gamma f) z, x-z\rangle \geq 0, \forall x \in F(S) \cap E P(F),
$$

which is the optimality condition for the minimization problem

$$
\min _{x \in F(S) \cap E P(F)} \frac{1}{2}\langle A x, x\rangle-h(x),
$$


where $h$ is a potential function for $\gamma f$.

A typical problem is to minimize a quadratic function over the set of the fixed points of a nonexpansive mappings on a real Hilbert space $H$ :

$$
\min \frac{1}{2}\langle A x, x\rangle-h(x)
$$

where $A$ is strongly positive linear bounded operator and $h$ is a potential function for $\gamma f$, i.e., $h^{\prime}(x)=\gamma f$, for all $x \in H$.

Let $A: H \rightarrow H$ be an inverse strongly monotone mapping and $F: C \times C \rightarrow \mathbb{R}$ be a bifunction. Then we consider the following GEPS

$$
\text { Find } \tilde{x} \in C \text { such that } F(\tilde{x}, y)+\langle A x, y-x\rangle \geq 0 \text {, for all } y \in C \text {. }
$$

The set of such $x \in C$ is denoted by $\operatorname{GEPS}(F, A)$, i.e.,

$$
\operatorname{GEPS}(F, A)=\{x \in C: F(x, y)+\langle A x, y-x\rangle \geq 0, \forall y \in C\} .
$$

To study the generalized equilibrium problem (1.1), let $F$ satisfies the following conditions:

(A1) $F(x, x)=0$, for all $x \in C$;

(A2) $F$ is monotone, i.e., $F(x, y)+F(y, x) \leq 0$ for all $x, y \in C$;

(A3) for each $x, y, z \in C$, $\lim \sup _{t \rightarrow 0^{+}} F(t z+(1-t) x, y) \leq F(x, y)$;

(A4) for each $x \in C y \mapsto F(x, y)$ is convex and weakly lower semi-continuous.

Recently, Kamraska and Wangkeeree [8] introduced a new iterative by viscosity approximation methods in a Hilbert space. To be more precisely, they proved the following result:

Theorem 1.1. Let $S=(T(s))_{s \geq 0}$ be a nonexpansive semigroup on a real Hilbert space H. Let $f: H \rightarrow H$ be an $\alpha$-contraction, $A: H \rightarrow H$ a strongly positive linear bounded self-adjoint operator with coefficient $\bar{\gamma}$. Let $\gamma$ be a real number such that $0<\gamma<\frac{\bar{\gamma}}{\alpha}$. Let $G: H \times H \rightarrow \mathbb{R}$ be a mapping satisfying hypotheses (A1)-(A4) and $\Psi: H \rightarrow H$ an inverse-strongly monotone mapping with coefficients $\delta>0$ such that $F(S) \cap G E P(G, \Psi) \neq \varnothing$. Let the sequences $\left\{x_{n}\right\},\left\{u_{n}\right\}$ and $\left\{y_{n}\right\}$ be generated by

$$
\left\{\begin{array}{l}
x_{1} \in H \text { chosen arbitrary, } \\
G\left(u_{n}, y\right)+\left\langle\Psi x_{n}, y-u_{n}\right\rangle+\frac{1}{r_{n}}\left\langle y-u_{n}, u_{n}-x_{n}\right\rangle, \\
y_{n}=\beta_{n} x_{n}+\left(1-\beta_{n}\right) \frac{1}{s_{n}} \int_{0}^{s_{n}} T(s) u_{n} d s, x_{n+1}=\alpha_{n} \gamma f\left(x_{n}\right)+\left(I-\alpha_{n} A\right) y_{n}, \forall n \geq 1 .
\end{array}\right.
$$


Under the certain appropriate conditions, they proved that the sequences $\left\{x_{n}\right\},\left\{u_{n}\right\}$ and $\left\{y_{n}\right\}$ is strongly convergent to $z$, which is a unique solution in $F(S) \cap G E P(G, \Psi)$ of the variational inequality

$$
\langle(\gamma f-A) z, p-z\rangle \leq 0, \forall p \in F(S) \cap G E P(G, \Psi) .
$$

The problem studied in this paper is formulated as follows (By intuition from [6], [7], [8]): Let $C_{1}$ and $C_{2}$ be closed convex subsets in $H$. Suppose that $F(x, y)$ be a bifunction satisfy conditions $(A 1)-(A 4)$ with $C$ replaced by $C_{1}$ and let $\{T(S): s \in[0, \infty)\}$ be a nonexpansive semigroup on $C_{2}$. Find an element

$$
x^{*} \in \bigcap_{i=1}^{k} \operatorname{GEPS}\left(F_{i}, \Psi_{i}\right) \bigcap F(S),
$$

where $\operatorname{GEPS}\left(F_{i}, \Psi_{i}\right)$ and $F(S)$ the set of solutions of an general equilibrium problem system(GEPS) involving by a bifunction $F_{k}\left(u_{n}^{(i)}, y\right)$ on $C_{1} \times C_{1}$ and the fixed point set of a nonexpansive semigroup $\{T(S): s \in[0, \infty)\}$ on a closed convex subset $C_{2}$, respectively.

\section{Preliminaries and Lemmas}

The following lemmas will be useful for proving the main results of this article. Let $A$ be a strongly positive linear bounded operator on $H$ : that is, there exists a constant $\bar{\gamma}>0$ such that

$$
\langle A x, x\rangle \geq \bar{\gamma}\|x\|^{2} \text {, for all } x \in H \text {. }
$$

Lemma 2.1 ([13]). Assume A is a strongly positive linear bounded operator on a Hilbert space $H$ with coefficient $\bar{\gamma}>0$ and $0<\rho<\|A\|^{-1}$. Then $\|I-\rho A\| \leq I-\rho \bar{\gamma}$.

Lemma 2.2 ([2]). Let $C$ be a nonempty closed convex subset of $H$ and $F: C \times C \rightarrow \mathbb{R}$ be a bifunction satisfying (A1)-(A4). Then, for any $r>0$ and $x \in H$ there exists $z \in C$ such that

$$
F(z, y)+\frac{1}{r}\langle y-z, z-x\rangle \geq 0, \forall y \in C .
$$

Further, define

$$
T_{r} x=\left\{z \in C: F(z, y)+\frac{1}{r}\langle y-z, z-x\rangle \geq 0, \forall y \in C\right\}
$$

for all $r>0$ and $x \in H$. Then

(a) $T_{r}$ is single-valued;

(b) $T_{r}$ is firmly nonexpansive, i.e., for any $x, y \in H$

$$
\left\|T_{r} x-T_{r} y\right\|^{2} \leq\left\langle T_{r} x-T_{r} y, x-y\right\rangle ;
$$

(c) $F\left(T_{r}\right)=G E P(F)$; 
(d) $\left\|T_{s} x-T_{r} x\right\| \leq \frac{s-r}{s}\left\|T_{s} x-x\right\|$;

(e) $G E P(F)$ is closed and convex.

Remark 2.3. It is clear that for any $x \in H$ and $r>0$, by Lemma 2.2(a), there exists $z \in H$ such that

$$
F(z, y)+\frac{1}{r}\langle y-z, z-x\rangle \geq 0 \text {, for all } y \in H
$$

Replacing $x$ with $x-r \Psi x$ in (2.1), we obtain

$$
F(z, y)+\langle\Psi x, y-z\rangle+\frac{1}{r}\langle y-z, z-x\rangle \geq 0 \text {, for all } y \in H
$$

Lemma 2.4 ([19]). Let $\left\{x_{n}\right\}$ and $\left\{y_{n}\right\}$ be bounded sequences in a Banach space E and $\left\{\beta_{n}\right\}$ be a sequence in $[0,1]$ with $0<\liminf _{n \rightarrow \infty} \beta_{n} \leq \limsup _{n \rightarrow \infty} \beta_{n}<1$. Suppose

$x_{n+1}=\left(1-\beta_{n}\right) y_{n}+\beta_{n} x_{n}$ for all integers $n \geq 1$ and $\limsup _{n \rightarrow \infty}\left(\left\|y_{n+1}-y_{n}\right\|-\left\|x_{n+1}-x_{n}\right\|\right) \leq 0$. Then $\lim _{n \rightarrow \infty}\left\|y_{n}-x_{n}\right\|=0$.

Lemma 2.5 ([18]). Let $C$ be a nonempty bounded closed convex subset of $H$ and let $S=\{T(s)$ : $s \in[0, \infty)\}$ be a nonexpansive semigroup on $C$. Then for any $h \in[0, \infty)$,

$$
\limsup _{t \rightarrow \infty}\left\|\frac{1}{t} \int_{0}^{t} T(s) x d s-T(h)\left(\frac{1}{t} \int_{0}^{t} T(s) x d s\right)\right\|=0,
$$

for $x \in C$ and $t>0$.

Lemma 2.6 ([23]). Assume $\left\{a_{n}\right\}$ is a sequence of nonnegative numbers such that

$$
a_{n+1} \leq\left(1-\alpha_{n}\right) a_{n}+\delta_{n}
$$

where $\left\{\alpha_{n}\right\}$ is a sequence in $(0,1)$ and $\left\{\delta_{n}\right\}$ is a sequence in real number such that

(i) $\lim _{n \rightarrow \infty} \alpha_{n}=0, \sum_{n=1}^{\infty} \alpha_{n}=\infty$;

(ii) $\limsup _{n \rightarrow \infty} \frac{\delta_{n}}{\alpha_{n}} \leq 0$ or $\sum_{n=1}^{\infty}\left|\delta_{n}\right|<\infty$;

Then $\lim _{n \rightarrow \infty} a_{n}=0$.

Lemma 2.7 ([4]). If $C$ is a closed convex subset of $H, T$ is a nonexpansive mapping on $C,\left\{x_{n}\right\}$ is a sequence in $C$ such that $x_{n} \rightarrow x \in C$ and $x_{n}-T x_{n} \rightarrow 0$, then $x-T x=0$ 


\section{Explicit viscosity iterative algorithm}

The viscosity method has been successfully applied to various problems coming from calculus of variations, minimal surface problems, plasticity theory and phase transition. It plays a central role too in the study of degenerated elliptic and parabolic second order equations [9], [11], [12]. First abstract formulation of the properties of the viscosity approximation have been given by Tykhonov [22] in 1963 when studying ill-posed problems (see [3] for details). The concept of viscosity solution for Hamilton-Jacobi equations, which plays a crucial role in control theory, game theory and partial differential equations has been introduced by Crandall and Lions [5]. In this section, we introduce a explicit viscosity iterative algorithm for finding a common element of the set of solution for an equilibrium problem involving a bifunction defined on a closed convex subset and the set of fixed points for a nonexpansive semigroup.

In this section, we introduce a new iterative for finding a common element of the set of solution for an equilibrium problem involving a bifunction defined on a closed convex subset and the set fixed points for a nonexpansive semigroup.

Theorem 3.1. Let $H$ be a real Hilbert space. Asuume that

- $C_{1}, C_{2}$ are two nonempty convex closed subsets $H$,

- $F_{1}, F_{2}, \ldots, F_{k}$ be bifunctions from $C_{1} \times C_{1}$ to $\mathbb{R}$ satisfying $(A 1)-(A 4)$,

- $\Psi_{1}, \Psi_{2}, \ldots, \Psi_{k}$ is $\mu_{i}$-inverse strongly monotone mapping on $H$,

- $f: H \rightarrow H$ is a $\rho$-contraction,

- A is a strongly positive linear bounded operator on $H$ with coefficient $\lambda$ and $0<\gamma<\frac{\lambda}{\rho}$,

- $F(S)=\{T(s): s \in[0, \infty)\}$ is a nonexpansive semigroup on $C_{2}$ such that $\bigcap_{i=1}^{k} F(S) \cap G E P S\left(F_{i}\right.$, $\left.\Psi_{i}\right) \neq \varnothing$,

- $\left\{x_{n}\right\}$ is a sequence generated in the following manner:

$$
\left\{\begin{array}{l}
x_{1} \in H, \text { and } u_{n}^{(i)} \in C_{1}, \\
F_{1}\left(u_{n}^{(1)}, y\right)+\left\langle\Psi_{1} x_{n}, y-u_{n}^{(1)}\right\rangle+\frac{1}{r_{n}}\left\langle y-u_{n}^{(1)}, u_{n}^{(1)}-x_{n}\right\rangle \geq 0, \text { for all } y \in C_{1}, \\
F_{2}\left(u_{n}^{(2)}, y\right)+\left\langle\Psi_{2} x_{n}, y-u_{n}^{(2)}\right\rangle+\frac{1}{r_{n}}\left\langle y-u_{n}^{(2)}, u_{n}^{(2)}-x_{n}\right\rangle \geq 0, \text { for all } y \in C_{1}, \\
\vdots \\
F_{k}\left(u_{n}^{(k)}, y\right)+\left\langle\Psi_{k} x_{n}, y-u_{n}^{(k)}\right\rangle+\frac{1}{r_{n}}\left\langle y-u_{n}^{(k)}, u_{n}^{(k)}-x_{n}\right\rangle \geq 0, \text { for all } y \in C_{1}, \\
\omega_{n}=\frac{1}{k} \sum_{i=1}^{k} u_{n}^{(i)}, \\
x_{n+1}=\alpha_{n} \gamma f\left(x_{n}\right)+\beta_{n} x_{n}+\left(\left(1-\beta_{n}\right) I-\alpha_{n} A\right) \frac{1}{t_{n}} \int_{0}^{t_{n}} T(s) P_{C_{2}} \omega_{n} d s,
\end{array}\right.
$$

where $\left\{\alpha_{n}\right\},\left\{\beta_{n}\right\}$ are the sequences in $[0,1]$ and $\left\{r_{n}\right\} \subset(0, \infty)$ is a real sequence which satisfy the following conditions: 
(C1) $\lim _{n \rightarrow \infty} \alpha_{n}=0, \sum_{n=1}^{\infty} \alpha_{n}=\infty$;

(C2) $0<\liminf _{n \rightarrow \infty} \beta_{n} \leq \limsup _{n \rightarrow \infty} \beta_{n}<1$;

(C3) $\lim _{n \rightarrow \infty}\left|r_{n+1}-r_{n}\right|=0$ and $0<b<r_{n}<a<2 \mu_{i}$ for $i \in\{1,2, \ldots, k\}$;

(C4) $\lim _{n \rightarrow \infty} t_{n}=\infty$, and sup $\left|t_{n+1}-t_{n}\right|$ is bounded.

Then

(i) the sequence $\left\{x_{n}\right\}$ is bounded;

(ii) $\lim _{n \rightarrow \infty}\left\|\Psi_{i} x_{n}-\Psi_{i} x^{*}\right\|=0$, for $i \in\{1,2, \ldots, k\}, x^{*} \in \bigcap_{i=1}^{k} \operatorname{GEPS}\left(F_{i}, \Psi_{i}\right) \cap F(S)$;

(iii) $\lim _{n \rightarrow \infty}\left\|x_{n}-\frac{1}{t_{n}} \int_{0}^{t_{n}} T(s) P_{C_{2}} \omega_{n} d s\right\|=0$ and $\lim _{n \rightarrow \infty}\left\|\omega_{n}-\frac{1}{t_{n}} \int_{0}^{t_{n}} T(s) P_{C_{2}} \omega_{n} d s\right\|=0$.

Proof. (i) By the same argument in [7, 10],

$$
\left\|\left(1-\beta_{n}\right) I-\alpha_{n} A\right\| \leq 1-\beta_{n}-\alpha_{n} \lambda .
$$

Let $q \in \bigcap_{i=1}^{k} F(S) \cap G E P S\left(F_{i}, \Psi_{i}\right)$. Observe that $I-r_{n} \Psi_{i}$ for any $i=1,2, \ldots, k$ is a nonexpansive mapping. Indeed, for any $x, y \in H$,

$$
\begin{aligned}
\left\|\left(I-r_{n} \Psi_{i}\right) x-\left(I-r_{n} \Psi_{i}\right) y\right\|^{2} & =\left\|(x-y)-r_{n}\left(\Psi_{i} x-\Psi_{i} y\right)\right\|^{2} \\
& =\|x-y\|^{2}-2 r_{n}\left\langle x-y, \Psi_{i} x-\Psi_{i} y\right\rangle+r_{n}^{2}\left\|\Psi_{i} x-\Psi_{i} y\right\|^{2} \\
& \leq\|x-y\|^{2}-r_{n}\left(2 \mu_{i}-r_{n}\right)\left\|\Psi_{i} x-\Psi_{i} y\right\|^{2} \\
& \leq\|x-y\|^{2} .
\end{aligned}
$$

So

$$
\left\|u_{n}^{(i)}-q\right\| \leq\left\|x_{n}-q\right\|
$$

and hence

$$
\left\|\omega_{n}-q\right\| \leq\left\|x_{n}-q\right\| .
$$

Thus

$$
\begin{aligned}
\left\|x_{n+1}-q\right\|= & \left\|\alpha_{n} \gamma f\left(x_{n}\right)+\beta_{n} x_{n}+\left(\left(1-\beta_{n}\right) I-\alpha_{n} A\right) \frac{1}{t_{n}} \int_{0}^{t_{n}} T(s) P_{C_{2}} \omega_{n} d s-q\right\| \\
\leq & \alpha_{n}\left\|\gamma f\left(x_{n}\right)-A q\right\|+\beta_{n}\left\|x_{n}-q\right\| \\
& \left.+\|\left(1-\beta_{n}\right) I-\alpha_{n} A\right)\|\| \frac{1}{t_{n}} \int_{0}^{t_{n}} T(s) P_{C_{2}} \omega_{n} d s-q \|
\end{aligned}
$$




$$
\begin{aligned}
\leq & \alpha_{n}\left\{\left\|\gamma f\left(x_{n}\right)-\gamma f(q)\right\|+\|\gamma f(q)-A q\|\right\}+\beta_{n}\left\|x_{n}-q\right\| \\
& +\left(1-\beta_{n}-\alpha_{n} \lambda\right) \frac{1}{t_{n}} \int_{0}^{t_{n}}\left\|T(s) P_{C_{2}} \omega_{n}-P_{C_{2}} q\right\| d s \\
\leq & \alpha_{n} \rho \gamma\left\|x_{n}-q\right\|+\alpha_{n}\|\gamma f(q)-A q\|+\beta_{n}\left\|x_{n}-q\right\| \\
& +\left(1-\beta_{n}-\alpha_{n} \lambda\right)\left\|\omega_{n}-q\right\| \\
\leq & \alpha_{n} \rho \gamma\left\|x_{n}-q\right\|+\alpha_{n}\|\gamma f(q)-A q\|+\beta_{n}\left\|x_{n}-q\right\| \\
& +\left(1-\beta_{n}-\alpha_{n} \lambda\right)\left\|x_{n}-q\right\| \\
= & \left(1-\alpha_{n}(\lambda-\gamma \rho)\right)\left\|x_{n}-q\right\|+\alpha_{n}\|\gamma f(q)-A q\| \\
\leq & \max \left\{\left\|x_{n}-q\right\|, \frac{\|\gamma f(q)-A q\|}{\lambda-\gamma \rho}\right\} .
\end{aligned}
$$

By induction

$$
\left\|x_{n}-q\right\| \leq \max \left\{\left\|x_{1}-q\right\|, \frac{\|\gamma f(q)-A q\|}{\lambda-\gamma \rho}\right\} .
$$

Therefore, the sequence $\left\{x_{n}\right\}$ is bounded and also $\left\{f\left(x_{n}\right)\right\},\left\{\omega_{n}\right\}$ and $\left\{\frac{1}{t_{n}} \int_{0}^{t_{n}} T(s) P_{C_{2}} \omega_{n} d s\right\}$ are bounded.

(ii) Note that $u_{n}^{(i)}$ can be written as $u_{n}^{(i)}=T_{r_{n}^{(i)}}\left(x_{n}-r_{n} \Psi_{i} x_{n}\right)$. By Lemma 2.2, for any $i=$ $1,2, \ldots, k$,

$$
\begin{aligned}
\left\|u_{n+1}^{(i)}-u_{n}^{(i)}\right\| \leq & \left\|T_{r_{n+1}^{(i)}}\left(I-r_{n+1} \Psi_{i}\right) x_{n+1}-T_{r_{n+1}^{(i)}}\left(I-r_{n} \Psi_{i}\right) x_{n}\right\| \\
& +\left\|T_{r_{n+1}^{(i)}}\left(I-r_{n} \Psi_{i}\right) x_{n}-T_{r_{n}^{(i)}}\left(I-r_{n} \Psi_{i}\right) x_{n}\right\| \\
\leq & \left\|\left(I-r_{n+1} \Psi_{i}\right) x_{n+1}-\left(I-r_{n} \Psi_{i}\right) x_{n}\right\| \\
& +\left\|T_{r_{n+1}^{(i)}}\left(I-r_{n} \Psi_{i}\right) x_{n}-T_{r_{n}^{(i)}}\left(I-r_{n} \Psi_{i}\right) x_{n}\right\| \\
\leq & \left\|x_{n+1}-x_{n}\right\|+\left|r_{n+1}-r_{n}\right|\left\|\Psi_{i} x_{n}\right\| \\
& +\frac{r_{n+1}-r_{n}}{r_{n+1}}\left\|T_{r_{n+1}^{(i)}}\left(I-r_{n} \Psi_{i}\right) x_{n}-T_{r_{n}^{(i)}}\left(I-r_{n} \Psi_{i}\right) x_{n}\right\| .
\end{aligned}
$$

Then

$$
\left\|u_{n+1}^{(i)}-u_{n}^{(i)}\right\| \leq\left\|x_{n+1}-x_{n}\right\|+2 M_{i}\left|r_{n+1}-r_{n}\right|
$$

where $\quad M_{i}=\max \left\{\sup \left\{\frac{\left\|T_{r_{n+1}^{(i)}}\left(I-r_{n} \Psi_{i}\right) x_{n}-T_{r_{n}^{(i)}}\left(I-r_{n} \Psi_{i}\right) x_{n}\right\|}{r_{n+1}}, \sup \left\{\left\|\Psi_{i} x_{n}\right\|\right\}\right\}\right.$.

Also

$$
\begin{aligned}
& \left\|\frac{1}{t_{n+1}} \int_{0}^{t_{n+1}} T(s) P_{C_{2}} \omega_{n+1} d s-\frac{1}{t_{n}} \int_{0}^{t_{n}} T(s) P_{C_{2}} \omega_{n} d s\right\| \\
= & \| \frac{1}{t_{n+1}} \int_{0}^{t_{n+1}}\left[T(s) \omega_{n+1}-T(s) \omega_{n}\right] d s+\left(\frac{1}{t_{n+1}}-\frac{1}{t_{n}}\right) \int_{0}^{t_{n}}\left[T(s) \omega_{n}-T(s) q\right] d s \\
& +\frac{1}{t_{n+1}} \int_{t_{n}}^{t_{n+1}}\left[T(s) \omega_{n}-T(s) q\right] d s \|
\end{aligned}
$$




$$
\leq\left\|\omega_{n+1}-\omega_{n}\right\|+\frac{2\left|t_{n+1}-t_{n}\right|}{t_{n+1}}\left\|\omega_{n}-q\right\| .
$$

Let $M=\frac{1}{k} \sum_{i=1}^{k} 2 M_{i}<\infty$, since

$$
\left\|\omega_{n+1}-\omega_{n}\right\| \leq \frac{1}{k} \sum_{i=1}^{k}\left\|u_{n+1}^{(i)}-u_{n}^{(i)}\right\| \leq\left\|x_{n+1}-x_{n}\right\|+M\left|r_{n+1}-r_{n}\right|,
$$

hence

$$
\begin{aligned}
& \left\|\frac{1}{t_{n+1}} \int_{0}^{t_{n+1}} T(s) P_{C_{2}} \omega_{n+1} d s-\frac{1}{t_{n}} \int_{0}^{t_{n}} T(s) P_{C_{2}} \omega_{n} d s\right\| \\
& \leq\left\|x_{n+1}-x_{n}\right\|+M\left|r_{n+1}-r_{n}\right|+\frac{2\left|t_{n+1}-t_{n}\right|}{t_{n+1}}\left\|\omega_{n}-q\right\| .
\end{aligned}
$$

Suppose $z_{n}=\frac{\alpha_{n} \gamma f\left(x_{n}\right)+\left(\left(1-\beta_{n}\right) I-\alpha_{n} A\right) \Lambda_{n}}{1-\beta_{n}}$, where $\Lambda_{n}:=\frac{1}{t_{n}} \int_{0}^{t_{n}} T(s) P_{C_{2}} \omega_{n} d s$. It follows from (3.3), (3.4)

$$
\begin{aligned}
\left\|z_{n+1}-z_{n}\right\|= & \| \frac{\alpha_{n+1} \gamma f\left(x_{n+1}\right)+\left(\left(1-\beta_{n+1}\right) I-\alpha_{n+1} A\right) \Lambda_{n+1}}{1-\beta_{n+1}} \\
& -\frac{\alpha_{n} \gamma f\left(x_{n}\right)+\left(\left(1-\beta_{n}\right) I-\alpha_{n} A\right) \Lambda_{n}}{1-\beta_{n}} \| \\
= & \| \frac{\alpha_{n+1} \gamma f\left(x_{n+1}\right)}{1-\beta_{n+1}}+\frac{\left(1-\beta_{n+1}\right) \Lambda_{n+1}}{1-\beta_{n+1}}-\frac{\alpha_{n+1} A \Lambda_{n+1}}{1-\beta_{n+1}} \\
& -\frac{\alpha_{n} \gamma f\left(x_{n}\right)}{1-\beta_{n}}-\frac{\left(1-\beta_{n}\right) \Lambda_{n}}{1-\beta_{n}}+\frac{\alpha_{n} A \Lambda_{n}}{1-\beta_{n}} \| \\
= & \left\|\frac{\alpha_{n+1}}{1-\beta_{n+1}}\left(\gamma f\left(x_{n+1}\right)-A \Lambda_{n+1}\right)+\frac{\alpha_{n}}{1-\beta_{n}}\left(A \Lambda_{n}-\gamma f\left(x_{n}\right)\right)+\left(\Lambda_{n+1}-\Lambda_{n}\right)\right\| \\
\leq & \frac{\alpha_{n+1}}{1-\beta_{n+1}}\left\|\gamma f\left(x_{n+1}\right)-A \Lambda_{n+1}\right\|+\frac{\alpha_{n}}{1-\beta_{n}}\left\|A \Lambda_{n}-\gamma f\left(x_{n}\right)\right\|+\left\|\Lambda_{n+1}-\Lambda_{n}\right\| \\
\leq & \frac{\alpha_{n+1}}{1-\beta_{n+1}}\left\|\gamma f\left(x_{n+1}\right)-A \Lambda_{n+1}\right\|+\frac{\alpha_{n}}{1-\beta_{n}}\left\|A \Lambda_{n}-\gamma f\left(x_{n}\right)\right\|+\left\|x_{n+1}-x_{n}\right\| \\
& +M\left|r_{n+1}-r_{n}\right|+\frac{2\left|t_{n+1}-t_{n}\right|}{t_{n+1}}\left\|\omega_{n}-q\right\| .
\end{aligned}
$$

(C1), (C3) and (C4) implies that

$$
\limsup _{n \rightarrow \infty}\left(\left\|z_{n+1}-z_{n}\right\|-\left\|x_{n+1}-x_{n}\right\|\right) \leq 0 .
$$

By Lemma 2.4

$$
\lim _{n \rightarrow \infty}\left\|z_{n}-x_{n}\right\|=0
$$

Consequently

$$
\lim _{n \rightarrow \infty}\left\|x_{n+1}-x_{n}\right\|=\lim _{n \rightarrow \infty}\left(1-\beta_{n}\right)\left\|z_{n}-x_{n}\right\|=0 .
$$

Moreover, for any $i \in\{1,2, \ldots, k\}$,

$$
\left\|u_{n}^{(i)}-q\right\|^{2} \leq\left\|\left(x_{n}-q\right)-r_{n}\left(\Psi_{i} x_{n}-\Psi_{i} q\right)\right\|^{2}
$$




$$
\begin{aligned}
& =\left\|x_{n}-q\right\|^{2}-2 r_{n}\left\langle x_{n}-q, \Psi_{i} x_{n}-\Psi_{i} q\right\rangle+r_{n}^{2}\left\|\Psi_{i} x_{n}-\Psi_{i} q\right\|^{2} \\
& \leq\left\|x_{n}-q\right\|^{2}-r_{n}\left(2 \mu_{i}-r_{n}\right)\left\|\Psi_{i} x_{n}-\Psi_{i} q\right\|^{2}
\end{aligned}
$$

and then

$$
\begin{aligned}
\left\|\omega_{n}-q\right\|^{2} & =\left\|\sum_{i=1}^{k} \frac{1}{k}\left(u_{n}^{(i)}-q\right)\right\|^{2} \leq \frac{1}{k} \sum_{i=1}^{k}\left\|u_{n}^{(i)}-q\right\|^{2} \\
& \left.\leq\left\|x_{n}-q\right\|^{2}-\frac{1}{k} \sum_{i=1}^{k} r_{n}\left(2 \mu_{i}-r_{n}\right) \| \Psi_{i} x_{n}-\Psi_{i} q\right) \|^{2} .
\end{aligned}
$$

By (3.6), we have

$$
\begin{aligned}
\left\|x_{n+1}-q\right\|^{2}= & \left\|\alpha_{n}\left(\gamma f\left(x_{n}\right)-A q\right)+\beta_{n}\left(x_{n}-q\right)+\left(\left(1-\beta_{n}\right) I-\alpha_{n} A\right)\left(\Lambda_{n}-q\right)\right\|^{2} \\
\leq & \alpha_{n}\left\|\gamma f\left(x_{n}\right)-A q\right\|^{2}+\beta_{n}\left\|x_{n}-q\right\|^{2}+\left(1-\beta_{n}-\alpha_{n} \lambda\right)\left\|\Lambda_{n}-q\right\|^{2} \\
\leq & \alpha_{n}\left\|\gamma f\left(x_{n}\right)-A q\right\|^{2}+\beta_{n}\left\|x_{n}-q\right\|^{2}+\left(1-\beta_{n}-\alpha_{n} \lambda\right)\left\|\omega_{n}-q\right\|^{2} \\
\leq & \alpha_{n}\left\|\gamma f\left(x_{n}\right)-A q\right\|^{2}+\beta_{n}\left\|x_{n}-q\right\|^{2}+\left(1-\beta_{n}-\alpha_{n} \lambda\right)\left\{\left\|x_{n}-q\right\|^{2}\right. \\
& \left.\left.-\frac{1}{k} \sum_{i=1}^{k} r_{n}\left(2 \mu_{i}-r_{n}\right) \| \Psi_{i} x_{n}-\Psi_{i} q\right) \|^{2}\right\} \\
\leq & \alpha_{n}\left\|\gamma f\left(x_{n}\right)-A q\right\|^{2}+\left\|x_{n}-q\right\|^{2} \\
& -\left(1-\beta_{n}-\alpha_{n} \lambda\right) \frac{1}{k} \sum_{i=1}^{k} r_{n}\left(2 \mu_{i}-r_{n}\right)\left\|\Psi_{i} x_{n}-\Psi_{i} q\right\|^{2},
\end{aligned}
$$

and hence

$$
\begin{aligned}
\left(1-\beta_{n}-\alpha_{n} \lambda\right) & \frac{1}{k} \sum_{i=1}^{k} b\left(2 \mu_{i}-a\right)\left\|\Psi_{i} x_{n}-\Psi_{i} q\right\|^{2} \\
& \leq \alpha_{n}\left\|\gamma f\left(x_{n}\right)-A q\right\|^{2}+\left\|x_{n}-q\right\|^{2}-\left\|x_{n+1}-q\right\|^{2} \\
& \leq \alpha_{n}\left\|\gamma f\left(x_{n}\right)-A q\right\|^{2}+\left\|x_{n+1}-x_{n}\right\|\left(\left\|x_{n+1}-q\right\|-\left\|x_{n}-q\right\|\right) .
\end{aligned}
$$

Since $\alpha_{n} \rightarrow 0$ and $\left\|x_{n+1}-x_{n}\right\| \rightarrow 0$, it follows that

$$
\lim _{n \rightarrow \infty}\left\|\Psi_{i} x_{n}-\Psi_{i} q\right\|=0, \forall i=1,2, \ldots, k .
$$

(iii) By Lemma 2.2, for any $i=1,2, \ldots, k$,

$$
\begin{aligned}
\left\|u_{n}^{(i)}-q\right\|^{2} \leq & \left\langle\left(I-r_{n} \Psi_{i}\right) x_{n}-\left(I-r_{n} \Psi_{i}\right) q, u_{n}^{(i)}-q\right\rangle \\
= & \frac{1}{2}\left\{\left\|\left(I-r_{n} \Psi_{i}\right) x_{n}-\left(I-r_{n} \Psi_{i}\right) q\right\|^{2}+\left\|u_{n, i}-q\right\|^{2}\right. \\
& \left.-\left\|\left(I-r_{n} \Psi_{i}\right) x_{n}-\left(I-r_{n} \Psi_{i}\right) q-\left(u_{n}^{(i)}-q\right)\right\|^{2}\right\} \\
\leq & \frac{1}{2}\left\{\left\|x_{n}-q\right\|^{2}+\left\|u_{n}^{(i)}-q\right\|^{2}-\left\|x_{n}-u_{n}^{(i)}-r_{n}\left(\Psi_{i} x_{n}-\Psi_{i} q\right)\right\|^{2}\right\} \\
= & \frac{1}{2}\left\{\left\|x_{n}-q\right\|^{2}+\left\|u_{n}^{(i)}-q\right\|^{2}-\left(\left\|x_{n}-u_{n}^{(i)}\right\|^{2}\right.\right. \\
& \left.-2 r_{n}\left\langle x_{n}-u_{n}^{(i)}, \Psi_{i} x_{n}-\Psi_{i} q\right\rangle+r_{n}^{2}\left\|\Psi_{i} x_{n}-\Psi_{i} q\right\|^{2}\right) .
\end{aligned}
$$


This implies

$$
\left\|u_{n}^{(i)}-q\right\|^{2} \leq\left\|x_{n}-q\right\|^{2}-\left\|x_{n}-u_{n}^{(i)}\right\|^{2}+2 r_{n}\left\|x_{n}-u_{n}^{(i)}\right\|\left\|\Psi_{i} x_{n}-\Psi_{i} q\right\|,
$$

and hence

$$
\begin{aligned}
\left\|\omega_{n}-q\right\|^{2} & =\left\|\sum_{i=1}^{k} \frac{1}{k}\left(u_{n}^{(i)}-q\right)\right\|^{2} \\
& \leq \frac{1}{k} \sum_{i=1}^{k}\left\|u_{n}^{(i)}-q\right\|^{2} \\
& \leq\left\|x_{n}-q\right\|^{2}-\frac{1}{k} \sum_{i=1}^{k}\left\|u_{n}^{(i)}-x_{n}\right\|^{2}+\frac{1}{k} \sum_{i=1}^{k} 2 r_{n}\left\|x_{n}-u_{n}^{(i)}\right\|\left\|\Psi_{i} x_{n}-\Psi_{i} q\right\| .
\end{aligned}
$$

Observe that

$$
\begin{aligned}
\left\|x_{n+1}-q\right\|^{2} \leq & \alpha_{n}\left\|\gamma f\left(x_{n}\right)-A q\right\|^{2}+\beta_{n}\left\|x_{n}-q\right\|^{2}+\left(1-\beta_{n}-\alpha_{n} \lambda\right)\left\|\omega_{n}-q\right\|^{2} \\
\leq & \alpha_{n}\left\|\gamma f\left(x_{n}\right)-A q\right\|^{2}+\beta_{n}\left\|x_{n}-q\right\|^{2}+\left(1-\beta_{n}-\alpha_{n} \lambda\right)\left\{\left\|x_{n}-q\right\|^{2}\right. \\
& \left.-\frac{1}{k} \sum_{i=1}^{k}\left\|u_{n}^{(i)}-x_{n}\right\|^{2}+\frac{1}{k} \sum_{i=1}^{k} 2 r_{n}\left\|x_{n}-u_{n}^{(i)}\right\|\left\|\Psi_{i} x_{n}-\Psi_{i} q\right\|\right\} .
\end{aligned}
$$

It follows that

$$
\begin{aligned}
\left(1-\beta_{n}-\alpha_{n} \lambda\right) \frac{1}{k} & \sum_{i=1}^{k}\left\|u_{n}^{(i)}-x_{n}\right\|^{2} \leq \alpha_{n}\left\|\gamma f\left(x_{n}\right)-A q\right\|^{2}+\left\|x_{n}-q\right\|^{2}-\left\|x_{n+1}-q\right\|^{2} \\
& +\left(1-\beta_{n}-\alpha_{n} \lambda\right) \frac{1}{k} \sum_{i=1}^{k} 2 r_{n}\left\|x_{n}-u_{n}^{(i)}\right\|\left\|\Psi_{i} x_{n}-\Psi_{i} q\right\| \\
& \leq \alpha_{n}\left\|\gamma f\left(x_{n}\right)-A q\right\|^{2}+\left\|x_{n+1}-x_{n}\right\|\left(\left\|x_{n}-q\right\|-\left\|x_{n+1}-q\right\|\right) \\
& +\left(1-\beta_{n}-\alpha_{n} \lambda\right) \frac{1}{k} \sum_{i=1}^{k} 2 r_{n}\left\|x_{n}-u_{n}^{(i)}\right\|\left\|\Psi_{i} x_{n}-\Psi_{i} q\right\| .
\end{aligned}
$$

Since $\alpha_{n} \rightarrow 0$ and $\left\|x_{n+1}-x_{n}\right\| \rightarrow 0$, we have

$$
\lim _{n \rightarrow \infty}\left\|u_{n}^{(i)}-x_{n}\right\|=0
$$

It is easy to prove

$$
\lim _{n \rightarrow \infty}\left\|\omega_{n}-x_{n}\right\|=0
$$

The definition of $\left\{x_{n}\right\}$ shows

$$
\begin{aligned}
\left\|\Lambda_{n}-x_{n}\right\| & \leq\left\|x_{n+1}-x_{n}\right\|+\left\|x_{n+1}-\Lambda_{n}\right\| \\
& \leq\left\|x_{n+1}-x_{n}\right\|+\left\|\alpha_{n} \gamma f\left(x_{n}\right)+\beta_{n} x_{n}+\left(\left(1-\beta_{n}\right) I-\alpha_{n} A\right) \Lambda_{n}-\Lambda_{n}\right\| \\
& \leq\left\|x_{n+1}-x_{n}\right\|+\alpha_{n}\left\|\gamma f\left(x_{n}\right)-A \Lambda_{n}\right\|+\beta_{n}\left\|x_{n}-\Lambda_{n}\right\| .
\end{aligned}
$$


That is

$$
\left\|\Lambda_{n}-x_{n}\right\| \leq \frac{1}{1-\beta_{n}}\left\|x_{n+1}-x_{n}\right\|+\frac{\alpha_{n}}{1-\beta_{n}}\left\|\gamma f\left(x_{n}\right)-A \Lambda_{n}\right\| .
$$

The condition (C1) together (3.5) implies that

$$
\lim _{n \rightarrow \infty}\left\|\Lambda_{n}-x_{n}\right\|=0
$$

Moreover, $\left\|\omega_{n}-\Lambda_{n}\right\| \leq\left\|\omega_{n}-x_{n}\right\|+\left\|x_{n}-\Lambda_{n}\right\|$, we get

$$
\lim _{n \rightarrow \infty}\left\|\Lambda_{n}-\omega_{n}\right\|=0
$$

Then,

$$
\begin{aligned}
& \lim _{n \rightarrow \infty}\left\|x_{n}-\frac{1}{t_{n}} \int_{0}^{t_{n}} T(s) P_{C_{2}} \omega_{n} d s\right\|=0, \\
& \lim _{n \rightarrow \infty}\left\|\omega_{n}-\frac{1}{t_{n}} \int_{0}^{t_{n}} T(s) P_{C_{2}} \omega_{n} d s\right\|=0 .
\end{aligned}
$$

Theorem 3.2. Suppose all assumptions of Theorem 3.1 are hold. Then the sequence $\left\{x_{n}\right\}$ is strongly convergent to a point $\bar{x}$, where $\bar{x} \in \bigcap_{i=1}^{k} F(S) \cap G E P S\left(F_{i}, \Psi_{i}\right)$, which solves the variational inequality

$$
\langle(A-\gamma f) \bar{x}, \bar{x}-x\rangle \leq 0 .
$$

Equivalently, $\bar{x}=P_{\bigcap_{i=1}^{k} F(S) \cap G E P S\left(F_{i}, \Psi_{i}\right)}(I-A+\gamma f)(\bar{x})$.

Proof. For all $x, y \in H$, we have

$$
\begin{aligned}
& \left\|P_{\bigcap_{i=1}^{k} F(S) \cap G E P S\left(F_{i}, \Psi_{i}\right)}(I-A+\gamma f)(x)-P_{\bigcap_{i=1}^{k} F(S) \cap G E P S\left(F_{i}, \Psi_{i}\right)}(I-A+\gamma f)(y)\right\| \\
\leq & \|(I-A+\gamma f)(x)-(I-A+\gamma f)(y)\| \\
\leq & \|I-A\|\|x-y\|+\gamma\|f(x)-f(y)\| \\
\leq & (1-\lambda)\|x-y\|+\gamma \rho\|x-y\| \\
= & (1-(\lambda-\gamma \rho))\|x-y\| .
\end{aligned}
$$

This implies that $P_{\cap_{i=1}^{k} F(S) \cap G E P S\left(F_{i}, \Psi_{i}\right)}(I-A+\gamma f)$ is a contraction of $H$ into itself. Since $H$ is complete, then there exists a unique element $\bar{x} \in H$ such that

$$
\bar{x}=P_{\bigcap_{i=1}^{k} F(S) \cap G E P S\left(F_{i}, \Psi_{i}\right)}(I-A+\gamma f)(\bar{x}) .
$$

Next, we prove

$$
\limsup _{n \rightarrow \infty}\left\langle(A-\gamma f) \bar{x}, \bar{x}-\frac{1}{t_{n}} \int_{0}^{t_{n}} T(s) P_{C_{2}} \omega_{n} d s\right\rangle \leq 0
$$


Let $\tilde{x}=P_{\bigcap_{i=1}^{k} F(S) \cap G E P S\left(F_{i}, \Psi_{i}\right)} x_{1}$, set

$$
\Sigma=\left\{\bar{y} \in C_{2}:\|\bar{y}-\tilde{x}\| \leq\left\|x_{1}-\tilde{x}\right\|+\frac{\|\gamma f(\tilde{x})-A \tilde{x}\|}{\lambda-\gamma \rho}\right\} .
$$

It is clear, $\Sigma$ is nonempty closed bounded convex subset of $C_{2}$ and $S=\{T(s): s \in[0, \infty)\}$ is a nonexpansive semigroup on $\Sigma$.

Let $\Lambda_{n}=\frac{1}{t_{n}} \int_{0}^{t_{n}} T(s) P_{C_{2}} \omega_{n} d s$, since $\left\{\Lambda_{n}\right\} \subset \Sigma$ is bounded, there is a subsequence $\left\{\Lambda_{n_{j}}\right\}$ of $\left\{\Lambda_{n}\right\}$ such that

$$
\limsup _{n \rightarrow \infty}\left\langle(A-\gamma f) \bar{x}, \bar{x}-\Lambda_{n}\right\rangle=\lim _{j \rightarrow \infty}\left\langle(A-\gamma f) \bar{x}, \bar{x}-\Lambda_{n_{j}}\right\rangle
$$

As $\left\{\omega_{n_{j}}\right\}$ is also bounded, there exists a subsequence $\left\{\omega_{n_{j_{l}}}\right\}$ of $\left\{\omega_{n_{j}}\right\}$ such that $\omega_{n_{j_{l}}} \rightarrow \xi$. Without loss of generality, let $\omega_{n_{j}} \rightarrow \xi$. From ( $i$ ii i) in Theorem 3.1, we have $\Lambda_{n_{j}} \rightarrow \xi$.

Since $\left\{\omega_{n}\right\} \subset C_{1}$ and $\left\{\Lambda_{n}\right\} \subset C_{2}$ and $C_{1}, C_{2}$ are two closed convex subsets in $H$, we obtain that $\xi \in C_{1} \cap C_{2}$.

Now, we prove the following items:

(i) $\xi \in F(S)=\bigcap_{s \geq 1} F(T(s))$.

Since $\left\{\Lambda_{n}\right\} \subset C_{2}$, we have

$$
\begin{aligned}
\left\|\Lambda_{n}-P_{C_{2}} \omega_{n}\right\| & =\left\|P_{C_{2}} \Lambda_{n}-P_{C_{2}} \omega_{n}\right\| \\
& \leq\left\|\Lambda_{n}-\omega_{n}\right\| .
\end{aligned}
$$

By ( $i$ i i) in Theorem 3.1, we have

$$
\lim _{n \rightarrow \infty}\left\|\Lambda_{n}-P_{C_{2}} \omega_{n}\right\|=0 .
$$

By using ( $i$ i i) in Theorem 3.1 and (3.15), we obtain

$$
\lim _{n \rightarrow \infty}\left\|\omega_{n}-P_{C_{2}} \omega_{n}\right\|=0 .
$$

This shows that the sequence $P_{C_{2}} \omega_{n_{j}} \rightarrow \xi$ as $j \rightarrow \infty$.

For each $h>0$, we have

$$
\begin{aligned}
\left\|T(h) P_{C_{2}} \omega_{n}-P_{C_{2}} \omega_{n}\right\| & \leq\left\|T(h) P_{C_{2}} \omega_{n}-T(h) \Lambda_{n}\right\|+\left\|T(h) \Lambda_{n}-\Lambda_{n}\right\|+\left\|\Lambda_{n}-P_{C_{2}} \omega_{n}\right\| \\
& \leq 2\left\|\Lambda_{n}-P_{C_{2}} \omega_{n}\right\|+\left\|T(h) \Lambda_{n}-\Lambda_{n}\right\| .
\end{aligned}
$$

The lemma 2.5 implies that

$$
\lim _{n \rightarrow \infty}\left\|T(h) \Lambda_{n}-\Lambda_{n}\right\|=0
$$


the equalities $(3.15,3.16)$ and (3.17)implies that

$$
\lim _{n \rightarrow \infty}\left\|T(h) P_{C_{2}} \omega_{n}-\omega_{n}\right\|=0 .
$$

Note that $F\left(T P_{C}\right)=F(T)$ for any mapping $T: C \rightarrow C$. The Lemma 2.7 implies that $\xi \in F\left(T(h) P_{C_{2}}\right)=F(T(h))$ for all $h>0$. This shows that $\xi \in F(S)$.

(ii) $\xi \in \bigcap_{i=1}^{k} \operatorname{GEPS}\left(F_{i}, \Psi_{i}\right)$.

Since $\left\{\omega_{n}\right\}$ is bounded and as respects (3.13), there exists a subsequence $\left\{\omega_{n_{j}}\right\}$ of $\left\{\omega_{n}\right\}$ such that $\omega_{n_{j}} \rightarrow \xi$. By intuition from [8],

$$
F_{i}\left(u_{n}^{(i)}, y\right)+\left\langle\Psi_{i} x_{n}, y-u_{n}^{(i)}\right\rangle+\frac{1}{r_{n}}\left\langle y-u_{n}^{(i)}, u_{n}^{(i)}-x_{n}\right\rangle \geq 0 \text {, for all } y \in C_{1} .
$$

By (A2), we have

$$
\left\langle\Psi_{i} x_{n}, y-u_{n}^{(i)}\right\rangle+\frac{1}{r_{n}}\left\langle y-u_{n}^{(i)}, u_{n}^{(i)}-x_{n}\right\rangle \geq F_{i}\left(y, u_{n}^{(i)}\right) .
$$

Substitute $n$ by $n_{j}$, we get

$$
\left\langle\Psi_{i} x_{n_{j}}, y-u_{n_{j}}^{(i)}\right\rangle+\left\langle y-u_{n_{j}}^{(i)}, \frac{u_{n_{j}}^{(i)}-x_{n_{j}}}{r_{n_{j}}}\right\rangle \geq F_{i}\left(y, u_{n_{j}}^{(i)}\right) .
$$

For $0<l \leq 1$ and $y \in C_{1}$, set $y_{l}=l y+(1-l) \xi$. We have $y_{l} \in C_{1}$ and

$$
\begin{aligned}
\left\langle y_{l}-u_{n_{j}}^{(i)}, \Psi_{i} y_{l}\right\rangle \geq & \left\langle y_{l}-u_{n_{j}}^{(i)}, \Psi_{i} y_{l}\right\rangle-\left\langle\Psi_{i} x_{n_{j}}, y_{l}-u_{n_{j}}^{(i)}\right\rangle \\
& -\left\langle y_{l}-u_{n_{j}}^{(i)}, \frac{u_{n_{j}}^{(i)}-x_{n_{j}}}{r_{n_{j}}}\right\rangle+F_{i}\left(y_{l}, u_{n_{j}}^{(i)}\right) \\
= & \left\langle y_{l}-u_{n_{j}}^{(i)}, \Psi_{i} y_{l}-\Psi_{i} u_{n_{j}}^{(i)}\right\rangle+\left\langle y_{l}-u_{n_{j}}^{(i)}, \Psi_{i} u_{n_{j}}^{(i)}-\Psi_{i} x_{n_{j}}\right\rangle \\
& -\left\langle y_{l}-u_{n_{j}}^{(i)}, \frac{u_{n_{j}}^{(i)}-x_{n_{j}}}{r_{n_{j}}}\right\rangle+F_{i}\left(y_{l}, u_{n_{j}}^{(i)}\right) .
\end{aligned}
$$

The condition (A4), monotonicity of $\Psi_{i}$ and (3.10) implies that $\left\langle y_{l}-u_{n_{j}}^{(i)}, \Psi_{i} y_{l}-\Psi_{i} u_{n_{j}}^{(i)}\right\rangle \geq 0$ and $\left\|\Psi_{i} u_{n_{j}}^{(i)}-\Psi_{i} x_{n_{j}}\right\| \rightarrow 0$ as $j \rightarrow \infty$. Hence

$$
\left\langle y_{l}-\xi, \Psi_{i} y_{l}\right\rangle \geq F_{i}\left(y_{l}, \xi\right) .
$$

Now, (A1) and (A4) together (3.19) show

$$
\begin{aligned}
0=F_{i}\left(y_{l}, y_{l}\right) & \leq l F_{i}\left(y_{l}, y\right)+(1-l) F_{i}\left(y_{l}, \xi\right) \\
& \leq l F_{i}\left(y_{l}, y\right)+(1-l)\left\langle y_{l}-\xi, \Psi_{i} y_{l}\right\rangle
\end{aligned}
$$




$$
=l F_{i}\left(y_{l}, y\right)+(1-l) l\left\langle y-\xi, \Psi_{i} y_{l}\right\rangle,
$$

which yields $F_{i}\left(y_{l}, y\right)+(1-l)\left\langle y-\xi, \Psi_{i} y_{l}\right\rangle \geq 0$.

By taking $l \rightarrow 0$, we have

$$
F_{i}(\xi, y)+\left\langle y-\xi, \Psi_{i} \xi\right\rangle \geq 0 .
$$

This shows $\xi \in G E P S\left(F_{i}, \Psi_{i}\right)$, for all $i=1,2, \ldots, k$. Then, $\xi \in \bigcap_{i=1}^{k} G E P S\left(F_{i}, \Psi_{i}\right)$.

Now, in view of (3.14), we see

$$
\limsup _{n \rightarrow \infty}\left\langle(A-\gamma f) \bar{x}, \bar{x}-\Lambda_{n}\right\rangle=\langle(A-\gamma f) \bar{x}, \bar{x}-\xi\rangle \leq 0 .
$$

Finally, we prove $\left\{x_{n}\right\}$ is strongly convergent to $\bar{x}$.

$$
\begin{aligned}
\left\|x_{n+1}-\bar{x}\right\|^{2}= & \left\|\alpha_{n} \gamma f\left(x_{n}\right)+\beta_{n} x_{n}+\left(\left(1-\beta_{n}\right) I-\alpha_{n} A\right) \Lambda_{n}-\bar{x}\right\|^{2} \\
= & \left\|\alpha_{n}\left(\gamma f\left(x_{n}\right)-A \bar{x}\right)+\beta_{n}\left(x_{n}-\bar{x}\right)+\left(\left(1-\beta_{n}\right) I-\alpha_{n} A\right)\left(\Lambda_{n}-\bar{x}\right)\right\|^{2} \\
= & \left\|\beta_{n}\left(x_{n}-\bar{x}\right)+\left(\left(1-\beta_{n}\right) I-\alpha_{n} A\right)\left(\Lambda_{n}-\bar{x}\right)\right\|^{2}+\alpha_{n}^{2}\left\|\gamma f\left(x_{n}\right)-A \bar{x}\right\|^{2} \\
& +2 \alpha_{n} \beta_{n}\left\langle x_{n}-\bar{x}, \gamma f\left(x_{n}\right)-A \bar{x}\right\rangle \\
& +2 \alpha_{n}\left\langle\left(\left(1-\beta_{n}\right) I-\alpha_{n} A\right)\left(\Lambda_{n}-\bar{x}\right), \gamma f\left(x_{n}\right)-A \bar{x}\right\rangle \\
\leq & \left\{\left(1-\beta_{n}-\alpha_{n} \lambda\right)\left\|\Lambda_{n}-\bar{x}\right\|+\beta_{n}\left\|x_{n}-\bar{x}\right\|\right\}^{2}+\alpha_{n}^{2}\left\|\gamma f\left(x_{n}\right)-A \bar{x}\right\|^{2} \\
& +2 \alpha_{n} \beta_{n} \gamma\left\langle x_{n}-\bar{x}, f\left(x_{n}\right)-f(\bar{x})\right\rangle+2 \alpha_{n} \beta_{n}\left\langle x_{n}-\bar{x}, \gamma f(\bar{x})-A \bar{x}\right\rangle \\
& +2\left(1-\beta_{n}\right) \gamma \alpha_{n}\left\langle\Lambda_{n}-\bar{x}, f\left(x_{n}\right)-f(\bar{x})\right\rangle \\
& +2\left(1-\beta_{n}\right) \alpha_{n}\left\langle\Lambda_{n}-\bar{x}, \gamma f(\bar{x})-A \bar{x}\right\rangle-2 \alpha_{n}^{2}\left\langle A\left(\Lambda_{n}-\bar{x}\right), \gamma f\left(x_{n}\right)-A \bar{x}\right\rangle .
\end{aligned}
$$

Consequently

$$
\begin{aligned}
\left\|x_{n+1}-\bar{x}\right\|^{2} \leq & \left\{\left(1-\alpha_{n} \lambda\right)^{2}+2 \rho \alpha_{n} \beta_{n} \gamma+2 \rho\left(1-\beta_{n}\right) \gamma \alpha_{n}\right\}\left\|x_{n}-\bar{x}\right\|^{2} \\
& +\alpha_{n}^{2}\left\|\gamma f\left(x_{n}\right)-A \bar{x}\right\|^{2}+2 \alpha_{n} \beta_{n}\left\langle x_{n}-\bar{x}, \gamma f(\bar{x})-A \bar{x}\right\rangle \\
& +2 \alpha_{n}\left(1-\beta_{n}\right)\left\langle\Lambda_{n}-\bar{x}, \gamma f(\bar{x})-A \bar{x}\right\rangle-2 \alpha_{n}^{2}\left\langle A\left(\Lambda_{n}-\bar{x}\right), \gamma f\left(x_{n}\right)-A \bar{x}\right\rangle \\
\leq & \left(1-2 \alpha_{n}(\lambda-\rho \gamma)\right)\left\|x_{n}-\bar{x}\right\|^{2}+\lambda^{2} \alpha_{n}^{2}\left\|x_{n}-\bar{x}\right\|^{2}+\alpha_{n}^{2}\left\|\gamma f\left(x_{n}\right)-A \bar{x}\right\|^{2} \\
& +2 \alpha_{n} \beta_{n}\left\langle x_{n}-\bar{x}, \gamma f(\bar{x})-A \bar{x}\right\rangle+2 \alpha_{n}\left(1-\beta_{n}\right)\left\langle\Lambda_{n}-\bar{x}, \gamma f(\bar{x})-A \bar{x}\right\rangle \\
& +2 \alpha_{n}^{2}\left\|A\left(\Lambda_{n}-\bar{x}\right)\right\|\left\|\gamma f\left(x_{n}\right)-A \bar{x}\right\| \\
= & \left(1-2 \alpha_{n}(\lambda-\rho \gamma)\right)\left\|x_{n}-\bar{x}\right\|^{2}+\alpha_{n}\left\{\alpha _ { n } \left(\lambda^{2}\left\|x_{n}-\bar{x}\right\|^{2}\right.\right. \\
& +\left\|\gamma f\left(x_{n}\right)-A \bar{x}\right\|^{2}+2\left\|A\left(\Lambda_{n}-\bar{x}\right)\right\|\left\|\gamma f\left(x_{n}\right)-A \bar{x}\right\| \\
& \left.+2 \beta_{n}\left\langle x_{n}-\bar{x}, \gamma f(\bar{x})-A \bar{x}\right\rangle+2\left(1-\beta_{n}\right)\left\langle\Lambda_{n}-\bar{x}, \gamma f(\bar{x})-A \bar{x}\right\rangle\right\} .
\end{aligned}
$$

Since $\left\{x_{n}\right\},\left\{f\left(x_{n}\right)\right\}$ and $\left\{\Lambda_{n}\right\}$ are bounded, one can take a constant $\Gamma>0$ such that

$$
\Gamma \geq \lambda^{2}\left\|x_{n}-\bar{x}\right\|^{2}+\left\|\gamma f\left(x_{n}\right)-A \bar{x}\right\|^{2}+2\left\|A\left(\Lambda_{n}-\bar{x}\right)\right\|\left\|\gamma f\left(x_{n}\right)-A \bar{x}\right\| .
$$


Let

$$
\Xi_{n}=2 \beta_{n}\left\langle x_{n}-\bar{x}, \gamma f(\bar{x})-A \bar{x}\right\rangle+2\left(1-\beta_{n}\right)\left\langle\Lambda_{n}-\bar{x}, \gamma f(\bar{x})-A \bar{x}\right\rangle+\Gamma \alpha_{n}
$$

Hence

$$
\left\|x_{n+1}-\bar{x}\right\|^{2} \leq\left(1-2 \alpha_{n}(\lambda-\rho \gamma)\right)\left\|x_{n}-\bar{x}\right\|^{2}+\alpha_{n} \Xi_{n} .
$$

With respect to (3.20), $\limsup _{n \rightarrow \infty} \Xi_{n} \leq 0$ and so all conditions of Lemma 2.6 are satisfied for (3.21). Consequently, the sequence $\left\{x_{n}\right\}$ is strongly convergent to $\bar{x}$.

As a result, by intuition from [8], the following mean ergodic theorem for a nonexpansive mapping in Hilbert space is proved.

Corollary 3.3. Suppose all assumptions of Theorem 3.1 are holds. Let $\left\{T^{i}\right\}$ be a family of nonexpansive mappings on $C_{1}$ for all $i=1,2, \ldots, k$ such that $\bigcap_{i=1}^{k} F\left(T^{i}\right) \cap G E P S\left(F_{i}, \Psi_{i}\right) \neq \varnothing$. Let $\left\{x_{n}\right\}$ and $\left\{u_{n}^{(i)}\right\} \subset C_{1}$ be sequences generated in the following manner:

$$
\left\{\begin{array}{l}
x_{1} \in H \text { choosen arbitrary, } \\
F_{1}\left(u_{n}^{(1)}, y\right)+\left\langle\Psi_{1} x_{n}, y-u_{n}^{(1)}\right\rangle+\frac{1}{r_{n}}\left\langle y-u_{n}^{(1)}, u_{n}^{(1)}-x_{n}\right\rangle \geq 0, \text { for all } y \in C_{1}, \\
F_{2}\left(u_{n}^{(2)}, y\right)+\left\langle\Psi_{2} x_{n}, y-u_{n}^{(2)}\right\rangle+\frac{1}{r_{n}}\left\langle y-u_{n}^{(2)}, u_{n}^{(2)}-x_{n}\right\rangle \geq 0, \text { for all } y \in C_{1}, \\
\vdots \\
F_{k}\left(u_{n}^{(k)}, y\right)+\left\langle\Psi_{k} x_{n}, y-u_{n}^{(k)}\right\rangle+\frac{1}{r_{n}}\left\langle y-u_{n}^{(k)}, u_{n}^{(k)}-x_{n}\right\rangle \geq 0, \text { for all } y \in C_{1}, \\
\omega_{n}=\frac{1}{k} \sum_{i=1}^{k} u_{n}^{(i)}, \\
x_{n+1}=\alpha_{n} \gamma f\left(x_{n}\right)+\beta_{n} x_{n}+\left(\left(1-\beta_{n}\right) I-\alpha_{n} A\right) \frac{1}{n+1} \sum_{i=0}^{n} P_{C_{2}} T^{i} \omega_{n},
\end{array}\right.
$$

where $\left\{\alpha_{n}\right\},\left\{\beta_{n}\right\}$ are the sequences in $[0,1]$ and $\left\{r_{n}\right\} \subset(0, \infty)$ is a real sequence. Suppose the following conditions are satisfied:

(C1) $\lim _{n \rightarrow \infty} \alpha_{n}=0, \sum_{n=1}^{\infty} \alpha_{n}=\infty$;

(C2) $0<\liminf _{n \rightarrow \infty} \beta_{n} \leq \limsup _{n \rightarrow \infty} \beta_{n}<1$;

(C3) $\lim _{n \rightarrow \infty}\left|r_{n+1}-r_{n}\right|=0$ and $0<b<r_{n}<a<2 \mu_{i}$ for $i \in\{1,2, \ldots, k\}$.

Then the sequence $\left\{x_{n}\right\}$ is strongly convergent to a point $\bar{x}$, where

$$
\bar{x}=P_{\bigcap_{i=1}^{k} F\left(T^{i}\right) \cap \operatorname{GEPS}\left(F_{i}, \Psi_{i}\right)}(I-A+\gamma f)(\bar{x}),
$$

is the unique solution of the variational inequality

$$
\langle(A-\gamma f) \bar{x}, \bar{x}-x\rangle \leq 0, \forall x \in \bigcap_{i=1}^{k} F\left(T^{i}\right) \cap \operatorname{GEPS}\left(F_{i}, \Psi_{i}\right) .
$$




\section{Application}

If $T(s)=T$ for all $s>0$ and $C_{1}=C_{2}=C$, then we have the following corollary.

Corollary 4.1. Let $H$ be a real Hilbert space, $F_{1}, F_{2}, \ldots, F_{k}$ be bifunctions from $C \times C$ to $\mathbb{R}$ satisfying $(A 1)-(A 4), \Psi_{1}, \Psi_{2}, \ldots, \Psi_{k}$ be $\mu_{i}$-inverse strongly monotone mapping on $H$, $A$ be a strongly positive linear bounded operator on $H$ with coefficient $\lambda$ and $0<\gamma<\frac{\lambda}{\rho}, f: H \rightarrow H$ be a $\rho$-contraction. Suppose that $T$ be a nonexpansive mapping on $C$ such that $\bigcap_{i=1}^{k} F(T) \cap$ $\operatorname{GEPS}\left(F_{i}, \Psi_{i}\right) \neq \varnothing$. Define the sequence $\left\{x_{n}\right\}$ as follows.

$$
\left\{\begin{array}{l}
x_{1} \in H, \text { and } u_{n}^{(i)} \in C, \\
F_{1}\left(u_{n}^{(1)}, y\right)+\left\langle\Psi_{1} x_{n}, y-u_{n}^{(1)}\right\rangle+\frac{1}{r_{n}}\left\langle y-u_{n}^{(1)}, u_{n}^{(1)}-x_{n}\right\rangle \geq 0, \text { for all } y \in C, \\
F_{2}\left(u_{n}^{(2)}, y\right)+\left\langle\Psi_{2} x_{n}, y-u_{n}^{(2)}\right\rangle+\frac{1}{r_{n}}\left\langle y-u_{n}^{(2)}, u_{n}^{(2)}-x_{n}\right\rangle \geq 0, \text { for all } y \in C, \\
\vdots \\
F_{k}\left(u_{n}^{(k)}, y\right)+\left\langle\Psi_{k} x_{n}, y-u_{n}^{(k)}\right\rangle+\frac{1}{r_{n}}\left\langle y-u_{n}^{(k)}, u_{n}^{(k)}-x_{n}\right\rangle \geq 0, \text { for all } y \in C, \\
\omega_{n}=\frac{1}{k} \sum_{i=1}^{k} u_{n}^{(i)}, \\
x_{n+1}=\alpha_{n} \gamma f\left(x_{n}\right)+\beta_{n} x_{n}+\left(\left(1-\beta_{n}\right) I-\alpha_{n} A\right) T P_{C} \omega_{n} d s,
\end{array}\right.
$$

where $\left\{\alpha_{n}\right\},\left\{\beta_{n}\right\} \subset[0,1]$ and $\left\{r_{n}\right\} \subset(0, \infty)$ are the sequences satisfying the conditions $(\mathrm{C} 1)-(\mathrm{C} 3)$ in Theorem 3.2. Then the sequence $\left\{x_{n}\right\}$ converges strongly to a point $\bar{x}$, where $\bar{x} \in \bigcap_{i=1}^{k} F(T) \cap$ $\operatorname{GEPS}\left(F_{i}, \Psi_{i}\right)$ solves the variational inequality

$$
\langle(A-\gamma f) \bar{x}, \bar{x}-x\rangle \leq 0 .
$$

We apply Theorem 3.2 for finding a common fixed point of a nonexpansive semigroup mappings and strictly pseudo-contractive mapping and inverse strongly monotone mapping. Recall that, a mapping $T: C \rightarrow C$ is called strictly pseudo-contractive if there exists $k$ with $0 \leq k \leq 1$ such that

$$
\|T x-T y\|^{2} \leq\|x-y\|^{2}+k\|(I-T) x-(I-T) y\|^{2}, \text { for all } x, y \in C .
$$

If $k=0$, then $T$ is nonexpansive. Put $J=I-T$, where $T: C \rightarrow C$ is a strictly pseudo-contractive mapping. $J$ is $\frac{1-k}{2}$-inverse strongly monotone and $J^{-1}(0)=F(T)$. Indeed, for all $x, y \in C$ we have

$$
\|(I-J) x-(I-J) y\|^{2} \leq\|x-y\|^{2}+k\|J x-J y\|^{2} .
$$

Also

$$
\|(I-J) x-(I-J) y\|^{2} \leq\|x-y\|^{2}+\|J x-J y\|^{2}-2\langle x-y, J x-J y\rangle .
$$

So, we have

$$
\langle x-y, J x-J y\rangle \geq \frac{1-k}{2}\|J x-J y\|^{2} .
$$


Corollary 4.2. Let $H$ be a real Hilbert space, $F_{1}, F_{2}, \ldots, F_{k}$ be bifunctions from $C \times C$ to $\mathbb{R}$ satisfying $(A 1)-(A 4), \Psi_{1}, \Psi_{2}, \ldots, \Psi_{k}$ be $\mu_{i}$-inverse strongly monotone mapping on $H$, $A$ be a strongly positive linear bounded operator on $H$ with coefficient $\lambda$ and $0<\gamma<\frac{\lambda}{\rho}, f: H \rightarrow H$ be a $\rho$-contraction. Suppose that $T: C \rightarrow H$ be a $k$-strictly pseudo-contractive mapping for some $0 \leq k<1$ such that $\bigcap_{i=1}^{k} F(T) \cap G E P S\left(F_{i}, \Psi_{i}\right) \neq \varnothing$. Define the sequence $\left\{x_{n}\right\}$ as follows.

$$
\left\{\begin{array}{l}
x_{1} \in H, \text { and } u_{n}^{(i)} \in C, \\
F_{1}\left(u_{n}^{(1)}, y\right)+\left\langle\Psi_{1} x_{n}, y-u_{n}^{(1)}\right\rangle+\frac{1}{r_{n}}\left\langle y-u_{n}^{(1)}, u_{n}^{(1)}-x_{n}\right\rangle \geq 0, \text { for all } y \in C, \\
F_{2}\left(u_{n}^{(2)}, y\right)+\left\langle\Psi_{2} x_{n}, y-u_{n}^{(2)}\right\rangle+\frac{1}{r_{n}}\left\langle y-u_{n}^{(2)}, u_{n}^{(2)}-x_{n}\right\rangle \geq 0, \text { for all } y \in C, \\
\vdots \\
F_{k}\left(u_{n}^{(k)}, y\right)+\left\langle\Psi_{k} x_{n}, y-u_{n}^{(k)}\right\rangle+\frac{1}{r_{n}}\left\langle y-u_{n}^{(k)}, u_{n}^{(k)}-x_{n}\right\rangle \geq 0, \text { for all } y \in C, \\
\omega_{n}=\frac{1}{k} \sum_{i=1}^{k} u_{n}^{(i)}, \\
x_{n+1}=\alpha_{n} \gamma f\left(x_{n}\right)+\beta_{n} x_{n}+\left(\left(1-\beta_{n}\right) I-\alpha_{n} A\right) P_{C} J \omega_{n} d s,
\end{array}\right.
$$

where $J: C \rightarrow H$ is a mapping defined by $J x=k x+(1-k) T x$ and $\left\{\alpha_{n}\right\},\left\{\beta_{n}\right\} \subset[0,1]$ and $\left\{r_{n}\right\} \subset$ $(0, \infty)$ are the sequences satisfying the conditions $(\mathrm{C} 1)-(\mathrm{C} 3)$ in Theorem 3.1 . Then the sequence $\left\{x_{n}\right\}$ is strongly convergent to a point $\bar{x}$, where $\bar{x} \in \bigcap_{i=1}^{k} F(T) \cap \operatorname{GEPS}\left(F_{i}, \Psi_{i}\right)$ solves the variational inequality

$$
\langle(A-\gamma f) \bar{x}, \bar{x}-x\rangle \leq 0 .
$$

Proof. Note that $S: C \rightarrow H$ is a nonexpansinve mapping and $F(T)=F(S)$. By Lemma 2.3 in [24] and Lemma 2.2 in [21], we have $P_{C} S: C_{2} \rightarrow C$ is a nonexpansive mapping and $F\left(P_{C} S\right)=$ $F(S)=F(S)$. Therefore, the result follows from Corollary 4.1 .

\section{Numerical Examples}

In this section, we show numerical examples which grantee the main theorem. The programming has been provided with Matlab according to the following algorithm.

Example 5.1. Suppose that $H=\mathbb{R}, C_{1}=[-1,1], C_{2}=[0,1]$ and

$$
F_{1}(x, y)=-3 x^{2}+x y+2 y^{2}, F_{2}(x, y)=-4 x^{2}+x y+3 y^{2}, F_{3}(x, y)=-5 x^{2}+x y+4 y^{2} .
$$

Also, we consider $\Psi_{1}(x)=x, \Psi_{2}=2 x$ and $\Psi_{3}(x)=\frac{x}{10}$. Suppose that $A=\frac{x}{10}, f(x)=\frac{x}{10}$ with coefficient $\gamma=1$ and $T(s)=e^{-s}$ is a nonexpansive semigroup on $C_{2}$. It is easy to check that $\Psi_{1}, \Psi_{2}, \Psi_{3}, A, f$ and $T(s)$ satisfy all conditions in Theorem 3.1. For each $y \in C_{1}$ there exists $z \in C_{1}$ such that

$$
F_{1}(z, y)+\left\langle\Psi_{1} x, y-z\right\rangle+\frac{1}{r}\langle y-z, z-x\rangle \geq 0
$$




$$
\begin{aligned}
& \Leftrightarrow-3 z^{2}+z y+2 y^{2}+x(y-z)+\frac{1}{r}(y-z)(z-x) \geq 0 \\
& \Leftrightarrow 2 r y^{2}+((r+1) z-(r-1) x) y-3 r z^{2}-x z r-z^{2}+z x \geq 0 .
\end{aligned}
$$

Set $G(y)=2 r y^{2}+((r+1) z-(r-1) x) y-3 r z^{2}-x z r-z^{2}+z x$. Then $G(y)$ is a quadratic function of $y$ with coefficients $a=2 r, b=(r+1) z-(r-1) x$ and $c=-3 r z^{2}-x z r-z^{2}+z x$. So

$$
\begin{aligned}
\Delta & =b^{2}-4 a c \\
& =[(r+1) z-(r-1) x]^{2}-8 r\left(-3 r z^{2}-x z r-z^{2}+z x\right) \\
& =x^{2}(r-1)^{2}+2 z x(r-1)(5 r+1)+z^{2}(5 r+1)^{2} \\
& =[(x(r-1)+z(5 r+1))]^{2} .
\end{aligned}
$$

Since $G(y) \geq 0$ for all $y \in C_{1}$, if and only if $\Delta=[(x(r-1)+z(5 r+1))]^{2} \leq 0$. Therefore, $z=\frac{1-r}{5 r+1} x$, which yields $T_{r_{n}^{(1)}}=u_{n}^{(1)}=\frac{1-r_{n}}{5 r_{n}+1} x_{n}$. By the same argument, for $F_{2}$ and $F_{3}$, one can conclude

$$
\begin{aligned}
& T_{r_{n}^{(2)}}=u_{n}^{(2)}=\frac{1-2 r_{n}}{7 r_{n}+1} x_{n}, \\
& T_{r_{n}^{(3)}}=u_{n}^{(3)}=\frac{10-r_{n}}{90 r_{n}+10} x_{n} .
\end{aligned}
$$

Then

$$
\begin{aligned}
\omega_{n} & =\frac{u_{n}^{(1)}+u_{n}^{(2)}+u_{n}^{(3)}}{3} \\
& =\frac{1}{3}\left[\frac{1-r_{n}}{5 r_{n}+1}+\frac{1-2 r_{n}}{7 r_{n}+1}+\frac{10-r_{n}}{90 r_{n}+10}\right] x_{n} .
\end{aligned}
$$

By choosing $r_{n}=\frac{n+8}{n}, t_{n}=n$, and $\alpha_{n}=\frac{9}{10 n}, \beta_{n}=\frac{2 n-1}{10 n-9}$, we have the following algorithm for the sequence $\left\{x_{n}\right\}$

$$
x_{n+1}=\frac{200 n^{2}-10 n-81}{100 n^{2}-90 n} x_{n}+\frac{800 n^{2}-890 n+81}{1000 n^{2}-900 n}\left(\frac{1-e^{-n}}{n}\right) \omega_{n} .
$$

Choose $x_{1}=1000$. By using MATLAB software, we obtain the following table and figure of the result.

Example 5.2. Theorem 3.2 can be illustrated by the following numerical example where the parameters are given as follows:

$$
\begin{gathered}
H=[-10,10], C_{1}=[-1,1], C_{2}=[0,1], A=I, f(x)=\frac{x}{5} \\
\Psi_{1}(x)=x, \Psi_{2}=2 x, \Psi_{3}(x)=\frac{x}{10}, \Psi_{4}(x)=3 x, \Psi_{5}(x)=4 x \\
\alpha_{n}=\frac{1}{2 n}, \beta_{n}=\frac{n}{2 n+1}, r_{n}=\frac{n+1}{n}
\end{gathered}
$$




\begin{tabular}{|c|c|c|c|c|c|}
\hline$n$ & $x_{n}$ & $n$ & $x_{n}$ & $n$ & $x_{n}$ \\
\hline 1 & 1000 & 11 & 0.001780587335 & 21 & 0.0000000003224455074 \\
2 & 1098.409511 & 12 & 0.0003806958425 & 22 & 0.00000000006766467135 \\
3 & 305.4917461 & 13 & 0.00008116570943 & 23 & 0.00000000001418205647 \\
4 & 73.2742215 & 14 & 0.00001726216226 & 24 & 0.000000000002969041259 \\
5 & 16.71274356 & 15 & 0.000003663196562 & 25 & 0.0000000000006208956961 \\
6 & 3.722532274 & 16 & 0.0000007758152752 & 26 & 0.000000000000007698339387 \\
7 & 0.8179356809 & 17 & 0.0000001640073634 & 27 & 0.0000000000001297089212 \\
8 & 0.1781302867 & 18 & 0.00000003461280635 & 28 & 0.00000000000002707018951 \\
9 & 0.03854703081 & 19 & 0.000000007293416021 & 29 & 0.000000000000005644205174 \\
10 & 0.008300951942 & 20 & 0.000000001534585156 & 30 & 0.000000000000001175770771 \\
\hline
\end{tabular}

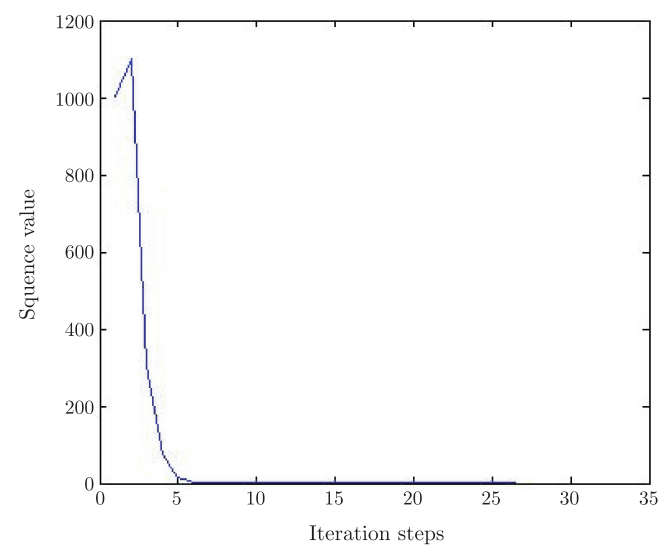

$$
T(s)=e^{-s}, \gamma=1, t_{n}=n
$$

Moreover,

$$
\begin{aligned}
& F_{1}(x, y)=-3 x^{2}+x y+2 y^{2}, F_{4}(x, y)=-6 x^{2}+x y+5 y^{2} \\
& F_{2}(x, y)=-4 x^{2}+x y+3 y^{2}, F_{5}(x, y)=-8 x^{2}+x y+7 y^{2} \\
& F_{3}(x, y)=-5 x^{2}+x y+4 y^{2} .
\end{aligned}
$$

By the same argument in Example 5.1, we compute $u_{n}^{(i)}$ for $i=1,2,3,4,5$ as follows:

$$
\begin{aligned}
& T_{r_{n}^{(1)}}=u_{n}^{(1)}=\frac{1-r_{n}}{5 r_{n}+1} x_{n}, \\
& T_{r_{n}^{(2)}}=u_{n}^{(2)}=\frac{1-2 r_{n}}{7 r_{n}+1} x_{n}, \\
& T_{r_{n}^{(3)}}=u_{n}^{(3)}=\frac{10-r_{n}}{90 r_{n}+10} x_{n}, \\
& T_{r_{n}^{(4)}}=u_{n}^{(4)}=\frac{1-3 r_{n}}{11 r_{n}+1} x_{n}, \\
& T_{r_{n}^{(5)}}=u_{n}^{(3)}=\frac{1-4 r_{n}}{15 r_{n}+1} x_{n} .
\end{aligned}
$$




\begin{tabular}{|c|c|c|c|c|c|}
\hline$n$ & $x_{n}$ & $n$ & $x_{n}$ & $n$ & $x_{n}$ \\
\hline 1 & 10 & 11 & 0.004288752976 & 21 & 0.000003269796382 \\
2 & 4.187523857 & 12 & 0.002073897432 & 22 & 0.000001606140362 \\
3 & 1.810011289 & 13 & 0.001005587742 & 23 & 0.0000007895721035 \\
4 & 0.8111798915 & 14 & 0.0004887121222 & 24 & 0.0000003884328747 \\
5 & 0.37213169 & 15 & 0.0002379837774 & 25 & 0.000000191218571 \\
6 & 0.173392393 & 16 & 0.0001160888527 & 26 & 0.00000009419138076 \\
7 & 0.08167872103 & 17 & 0.00005671409128 & 27 & 0.00000004642361668 \\
8 & 0.03878661445 & 18 & 0.00002774424594 & 28 & 0.00000002289261628 \\
9 & 0.0185321898 & 19 & 0.00001358854452 & 29 & 0.0000000112944317 \\
10 & 0.008897703796 & 20 & 0.000006662503243 & 30 & 0.00000000557482625 \\
\hline
\end{tabular}

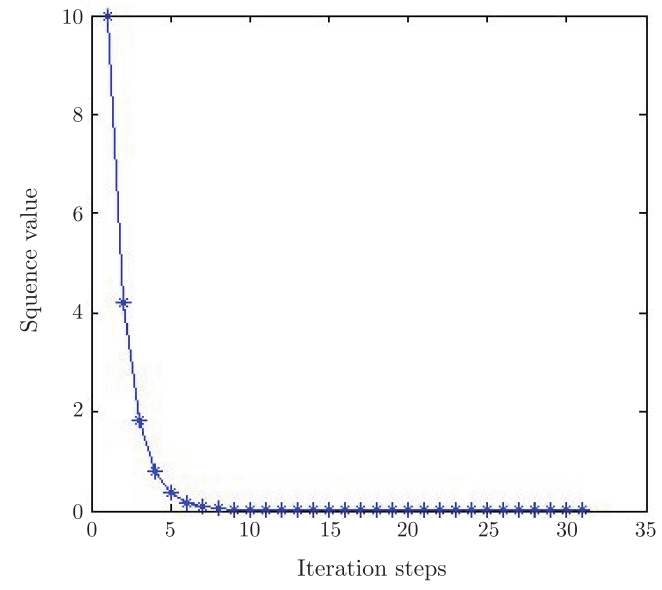

Then

$$
\begin{aligned}
\omega_{n} & =\frac{u_{n}^{(1)}+u_{n}^{(2)}+\ldots+u_{n}^{(5)}}{5} \\
& =\frac{1}{5}\left[\frac{1-r_{n}}{5 r_{n}+1}+\frac{1-2 r_{n}}{7 r_{n}+1}+\frac{10-r_{n}}{90 r_{n}+10}+\frac{1-3 r_{n}}{11 r_{n}+1}+\frac{1-4 r_{n}}{15 r_{n}+1}\right] x_{n} .
\end{aligned}
$$

Choose $x_{1}=10$. The detailed results of proposed iterative in Theorem 3.2 are presented in the following table and figure.

Example 5.3. Let

$$
\begin{gathered}
H=[-10,10], C_{1}=[0,1], C_{2}=[-1,1], A=\frac{x}{10}, f(x)=\frac{x}{10} \\
\Psi_{1}(x)=\Psi_{2}(x)=0, \Psi_{3}(x)=x, \Psi_{4}(x)=2 x, \Psi_{5}(x)=\frac{x}{10} \\
\Psi_{6}(x)=3 x, \Psi_{7}(x)=4 x \\
\alpha_{n}=\frac{1}{n}, \beta_{n}=\frac{n}{3 n+1}, r_{n}=\frac{n+1}{n}
\end{gathered}
$$




$$
T(s)=e^{-s}, \gamma=1, t_{n}=n
$$

Moreover,

$$
\begin{aligned}
& F_{1}(x, y)=\left(1-x^{2}\right)(x-y), F_{5}(x, y)=-5 x^{2}+x y+4 y^{2} \\
& F_{2}(x, y)=-x^{2}(x-y)^{2}, F_{6}(x, y)=-6 x^{2}+x y+5 y^{2} \\
& F_{3}(x, y)=-3 x^{2}+x y+2 y^{2}, F_{7}(x, y)=-8 x^{2}+x y+7 y^{2} \\
& F_{4}(x, y)=-4 x^{2}+x y+3 y^{2} .
\end{aligned}
$$

By the same argument in Example 5.1, we compute $u_{n}^{(i)}$ for $i=1,2$ as follows: For any $y \in C_{1}$ and $r>0$, we have

$$
F(z, y)+\frac{1}{r}\langle y-z, z-x\rangle \geq 0 \Leftrightarrow(y-z)\left(r z^{2}+z-r-x\right) \geq 0 .
$$

This implies that $r z^{2}+z-r-x=0$. Therefore, $z=\frac{-1+\sqrt{1+4 r(r+x)}}{2 r}$ which yields $T_{r_{n}^{(1)}}=\frac{-1+\sqrt{1+4 r_{n}\left(r_{n}+x_{n}\right)}}{2 r_{n}}$. Also, we have

$$
F(z, y)+\frac{1}{r}\langle y-z, z-x\rangle \geq 0 \Leftrightarrow-r z^{2} y^{2}+\left(2 r z^{3}+z-x\right) y-r z^{4}-z^{2}+z x \geq 0 .
$$

Set $J(y)=-r z^{2} y^{2}+\left(2 r z^{3}+z-x\right) y-r z^{4}-z^{2}+z x$. Then $J(y)$ is a quadratic function of $y$ with coefficients $a=-r z^{2}, b=2 r z^{3}+z-x$ and $c=-r z^{4}-z^{2}+z x$. So

$$
\begin{aligned}
\Delta & =\left[2 r z^{3}+z-x\right]^{2}+4 r z^{2}\left(-r z^{4}-z^{2}+z x\right) \\
& =(z-x)^{2} .
\end{aligned}
$$

Since $J(y) \geq 0$ for all $y \in H$, if and only if $\Delta=(z-x)^{2}=0$. Therefore, $z=T_{r_{n}^{(2)}}=x$. Then

$$
\begin{aligned}
& T_{r_{n}^{(1)}}=u_{n}^{(1)}=\frac{-1+\sqrt{1+4 r_{n}\left(r_{n}+x_{n}\right)}}{2 r_{n}}, \\
& T_{r_{n}^{(2)}}=u_{n}^{(2)}=x_{n}, \\
& T_{r_{n}^{(3)}}=u_{n}^{(3)}=\frac{1-r_{n}}{5 r_{n}+1} x_{n}, \\
& T_{r_{n}^{(4)}}=u_{n}^{(4)}=\frac{1-2 r_{n}}{7 r_{n}+1} x_{n}, \\
& T_{r_{n}^{(5)}}=u_{n}^{(5)}=\frac{10-r_{n}}{90 r_{n}+10} x_{n}, \\
& T_{r_{n}^{(6)}}=u_{n}^{(6)}=\frac{1-3 r_{n}}{11 r_{n}+1} x_{n}, \\
& T_{r_{n}^{(7)}}=u_{n}^{(7)}=\frac{1-4 r_{n}}{15 r_{n}+1} x_{n} .
\end{aligned}
$$




\begin{tabular}{|c|c|c|c|c|c|}
\hline$n$ & $x_{n}$ & $n$ & $x_{n}$ & $n$ & $x_{n}$ \\
\hline 1 & 10 & 11 & 0.0239156544 & 21 & 0.00001930391707 \\
2 & 7.414282455 & 12 & 0.01166047726 & 22 & 0.000009519419651 \\
3 & 5.25738677 & 13 & 0.005695329069 & 23 & 0.000004697209017 \\
4 & 3.188403646 & 14 & 0.002786256225 & 24 & 0.000002319056949 \\
5 & 1.720431319 & 15 & 0.001365047089 & 25 & 0.000001145527389 \\
6 & 0.8702902283 & 16 & 0.000669619601 & 26 & 0.0000005661151488 \\
7 & 0.4279164132 & 17 & 0.0003288520367 & 27 & 0.0000002798942327 \\
8 & 0.2082805371 & 18 & 0.0001616632095 & 28 & 0.000000138439269 \\
9 & 0.1011375703 & 19 & 0.00007954524563 & 29 & 0.00000006849968096 \\
10 & 0.04914122712 & 20 & 0.00003917150569 & 30 & 0.00000003390554052 \\
\hline
\end{tabular}

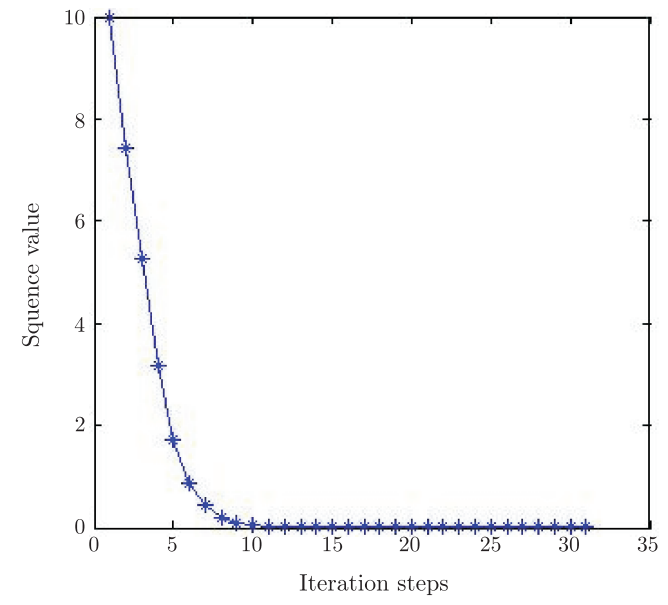

Then

$$
\omega_{n}=\frac{u_{n}^{(1)}+u_{n}^{(2)}+\ldots+u_{n}^{(7)}}{7}
$$

We have

$$
x_{n+1}=\frac{10 n^{2}+3 n+1}{30 n^{2}-10 n} x_{n}+\frac{20 n^{2}+7 n-1}{30 n^{2}+10 n}\left(\frac{1-e^{-n}}{n}\right) \omega_{n} .
$$

Choose $x_{1}=10$. The detailed results of proposed iterative in Theorem 3.2 are presented in the following table and figure.

\section{References}

[1] E. F. Browder, Fixed point theorems for nonexpansive mappings in Hilbert spaces, Proceed. Nat. Acad. Sci., 53 (1965), 1272-1276.

[2] P. Combettes and A. Histoaga, Equilibrium programming in Hilbert spaces, J. Nonlinear Convex Anal., 6 (2005), 117-136. 
[3] A. L. Dontchev and T. Zolezzi, Well-posed optimization problems, Lecture Notes in Mathematics, 1543 (1993), Springer-Verlag.

[4] K. Goebel and W. A. Kirk, Topics in metric fixed point theory, Vol 28 of Cambidge Studies in Advanced Mathematics, Cambridge University Press, Cambridge, U.K (1995).

[5] M. G. Crandall and P. L. Lions, Viscosity solution of Hamilton-Jacobi equations, Trans. Amer. Math. Soc., 277 (1983), 1-42.

[6] Z. He and W. S. Du, Strong convergence theorems for equilibrium problems and fixed point peoblems: A new iterative method, some comments and applications, Fixed Point Theroy Appl., Article ID. 33 (2011), 15 pages.

[7] T. Jitpeera, P. Kachang and P. Kumam, A viscosity of Ceasaro mean approximation methods for a mixed equilibrium, varational inequalities, and fixed point problems, Fixed Point Theroy Appl., Article ID. 945051 (2011), 24 pages.

[8] U. Kamraska and R. Wangkeeree, Generalized equilibrium problems and fixed point problems for nonexpansive semigroups in Hilbert spaces, J. Glob. Optim., 51 (2011), 689-714.

[9] R. V. Kohn and P. Sternberg, Local minimizers and singular perturbations, Proc. Royal Soc. Edinburgh, Sect. A, 111 (1989), 64-84.

[10] P. Kumam and P. Kachang, A viscosity of extragradient approximation method for finding equilibirum problem, varational inequlities and fixed point problems for nonexpansive mappings, Nonlinear Anal. Hybrid Syst., 3(2009), 475-486.

[11] O. A. Ladyzenskaya and N. N. Uralceva, Local estimates for gradients of solutions of uniformly elliptic and parabolic equations, Com. Pure Appl. Math., 18 (1970), 677-703.

[12] J. L. Lions, Quelques methodes de resolution des probl'emes aux limites nonlineaires, Dunod et GauthiersVillars, Paris, 1969.

[13] G. Marino and H. K. Xu, A genaral iterative method for nonexpansive mappings in Hilbert spaces, J. Math. Anal. Appl., 318 (2006), 43-52.

[14] K. Nakajo, W. Takahashi, Strong and weak convergence theorems by an improved splitting method, Comm. Appl. Nonlinear Anal. 9 (2002), 99-107.

[15] Z. Opial, Weak convergence of successive approximations for nonexpansive mappings, Bull. Amer. Math. Soc., 73 (1967), 591-597.

[16] S. Plubtieng and R. Punpaeng, A general iterative methods for equilibrium problems and fixed point problems in Hilbert spaces, J. Math. Appl., 336 (2007), 455-469.

[17] S. Plubtieng and R. Punpaeng, Fixed point solutions of variational inequalities for nonexpansive semigroups in Hilbert spaces, Math. Comput. Modelling, 48 (2008), 279-286.

[18] T. Shimizu and W. Takahashi, Strong convergence to common fixed points of nonexpansive mappings, J. Math. Anal. Appl., 211 (1997), 71-83.

[19] T. Suzuki, Strong convergence of Krasnoselkii and Mann's type sequences for one-parameter nonexpansive semigroups without Bochner integrals, J. Math. Anal. Appl., 305 (2005), 227-239.

[20] W. Takahashi, Nonlinear complemetarity problem and systems of convex inequalities, J. Optim. Theory Appl., 24 (1978), 493-503.

[21] K. K. Tan, H. K. Xu, The nonlinear ergodic theorem for asymptotically nonexapansive mappings in Banach spaces, Proc. Amer. Math. Soc., 114 (1992), 309-404.

[22] A. Tychonov, Solution of incorrectly formulated problems and the regularization method, Soviet Math. Dokl., 4 (1963), 1035-1038.

[23] H. K. Xu, Viscosity approximation methods for nonexpansive mappings, J. Math. Anal. Appl., 298 (2004), 279291.

[24] H. Zhou, Convergence theorems of fixed points for $k$-strict pseudo-contractions in Hilbert spaces, Nonlinear Anal., 69 (2008), 456-462.

Department of Mathematics, Ashtian Branch, Islamic Azad University, P.O. Box 39618-13347, Ashtian, Iran.

E-mail: sahebi@mail.aiau.ac.ir

E-mail: ebrahimi@mail.aiau.ac.ir 